\title{
Densidade Microvascular No CARCINOMA DE LíNGUA
}

\author{
All amar, Alan f. Giovanini, marilene P. Rosa, Hitomi O. Yamassaki, Marcos b. de Carvalho, abräo Rapoport*. \\ Trabalho realizado nos Serviços de Cirurgia de Cabeça e Pescoço e \\ Anatomia Patológica do Hospital Heliópolis, S. Paulo, SP.
}

RESUMO - OBJETIVO. Avaliar a densidade microvascular no carcinoma epidermóide de língua oral, no sítio primário e em suas metástases linfáticas.

MÉtODos. Foram avaliados retrospectivamente 30 pacientes com carcinoma epidermóide restrito à língua oral, submetidos a tratamento cirúrgico incluindo esvaziamento cervical. A densidade microvascular foi avaliada por imunohistoquímica empregando 0 anticorpo anti-CD34 e quantificada à microscopia óptica, no tumor primário e em suas metástases linfonodais. Foi avaliada a relação entre a densidade microvascular, as variáveis clínicas e histológicas e 0 prognóstico.

RESULTADOS. A densidade microvascular apresentou media- na de 15,4 vasos/campo $(5,5$ a 25,3$)$ nos tumores primários e 16,4 vasos/campo (12 a 32,2) nas metástases linfáticas. Foi observada uma relação inversa entre a densidade microvascular no tumor primário e na respectiva metástase linfática $(r=-0,68$ e $p=0,04)$. A densidade microvascular não apresentou relação com outras variáveis histológicas ou com 0 prognóstico.

CONCLUSÃo. Há uma relação inversa entre a densidade microvascular no sítio primário e na metástase linfonodal, sugerindo um controle regional ou sistêmico da angiogênese.

UNITERMOS: Câncer. Angiogênese. Metástase. Linfonodo. Língua.

\section{INTRODUÇÃO}

Os carcinomas epidermóides da língua são as neoplasias malignas mais freqüentes na boca. Quando operados em estádio avançado, esses tumores apresentam maior índice de margens comprometidas e, nestas circunstâncias, menor resposta ao tratamento radioterápico adjuvante ${ }^{1,2}$. No momento, não existem meios de identificar os pacientes que, ainda que estejam com doença em estádio inicial, precisam de tratamento mais agressivo, ou aqueles com doença em estádio avançado que não se beneficiam com as alternativas terapêuticas disponíveis. Aneoangiogêneseé um processo essencial para o desenvolvimento tumoral e tem sido relacionada com a capacidade de metastatização e o prognóstico em neoplasias malignas de diversos sítios anatômicos. Embora a metastatização seja a característica mais marcante das neoplasias malignas, os resultados de diferentes estudos realizados em carcinomas de boca têm se

*Correspondencia:

Praça Amadeu Amaral, 47 - cj. 82 Paraíso - CEP 01327-010 - São Paulo - SP

E-mail: cpgcp.hosphel@attglobal.net mostrado conflitantes quanto à importância da densidade microvascular e sugerem que 0 método, desenvolvido em outras neoplasias, necessita de adaptação $0^{4,5,6}$. Este estudo tem por objetivo avaliar a densidade da neoangiogênese nos carcinomas epidermóides de língua, (tumor primário e metástases linfonodais), relacionando estes achados com a presença de metástase ou recidiva.

\section{Métodos}

Foram revisados os prontuários de 104 pacientes com carcinoma epidermóide de língua oral submetidos a tratamento cirúrgico no Hospital Heliópolis entre 1979 e 1998. Todos os pacientes haviam sido submetidos ao esvaziamento cervical. Foram selecionados os 30 pacientes que tiveram seguimento superior a 24 meses (exceto dois pacientes com seguimento entre 18 e 24 meses), cujos blocos parafinizados apresentavam condições adequadas para a revisão histológica e realização de imuno-histoquímica. A radioterapia adjuvante foi empregada nos pacientes que apresentaram margens cirúrgicas comprometidas ou linfonodos metastáticos confirmados histologicamente.
Quanto ao sexo, 26 pacientes eram masculinos e quatro femininos. A idade média foi de 54 anos. Dois casos apresentavam estadiamento $\mathrm{TI}, 14$ casos T2, 13 casos $\mathrm{T} 3$ e I caso T4.

O material foi submetido à revisão anatomopatológica convencional considerando os seguintes fatores: grau de diferenciação, padrão de invasão, presença de necrose, infiltrado inflamatório e desmoplasia. Nesta análise foram selecionados os cortes mais significativos do tumor primário e de suas metástases linfonodais, para realização de reação imuno-histoquímica com anticorpo antiCD34 (Figura I). Os cortes histológicos com $3 \mathrm{~mm}$ de espessura passaram por processo de desparafinização. $O$ processo de recuperação antigênica empregou ácido cítrico $10 \mathrm{mM}(\mathrm{pH} \mathrm{6,0)} \mathrm{e} \mathrm{forno} \mathrm{micro-}$ ondas em três sessões de cinco minutos. $A$ incubação das lâminas com o anticorpo primário foi realizada em câmara úmida, à temperatura ambiente, durante 60 minutos. O anticorpo anti-CD34 (fornecido pela Dako A/S - Dinamarca) foi diluído em BSA na proporção de I:50. Foi realizada a 
incubação com o anticorpo secundário e terciário do complexo streptavidinabiotina, "kit LSAB" (DAKO número KO 690) durante 20 minutos, revelados pela diaminobenzidina e contra-corados por hematoxilina de Mayer.

A mensuração da densidade microvascular foi realizada em três áreas de maior imuno-marcação: hot spots intra-tumorais. A contagem de vasos foi realizada à microscopia de luz com magnificação de 250 vezes, após sua individualização pelo software Imagelab-Softium.

A análise estatística empregou o teste exato de Fisher para avaliar a diferença entre variáveis qualitativas, 0 teste de Mann-Whitney para variáveis quantitativas e 0 teste de Spearman para correlação entre variáveis. Foram considerados significativas as diferenças com erro alfa inferior a $5 \%$.

Este estudo foi realizado com apoio financeiro da FAPESP - Fundação de Amparo à Pesquisa do Estado de São Paulo.

\section{RESULTADOS}

A densidade microvascular apresentou mediana de I5,4 vasos/campo $(5,5$ a 25,3$)$ nos tumores primários e 16,4 vasos/campo $(12$ a 32,2$)$ nas metástases linfáticas (Figura 2). Avaliando a densidade microvascular no sítio primário e na respectiva metástase, foi observada uma relação inversa nestes dois sítios, $r=-0,68$ e $p=0,04$, sendo que a densidade vascular mediana no tumor primário se relacionou com densidade vascular mediana nas metástases (Figura 3).

Não houve relação significativa entre a densidade microvascular no sítio primário e as variáveis histológicas avaliadas rotineiramente, ou seja, grau de diferenciação, padrão de invasão, infiltrado inflamatório, necrose edesmoplasia (Tabela I).

As lesões iniciais ( $\mathrm{Tl}$ e $\mathrm{T} 2$ ) apresentaram densidade microvascular mediana de 15,3 vasos/campo (II a 20,6) e as lesões avançadas (T3 e T4) apresentaram mediana de 16 vasos/ campo (5,5 a 25,3), $p=0,8 \mathrm{I}$ (Figura 4).

Os tumores primários com maior densidade microvascular não apresentavam metástases linfáticas. Os casos sem metástases (pN0) apresentaram mediana de 16 vasos/
Figura I - Vasos marcados pelo anticorpo anti-CD34. Aumento 400X

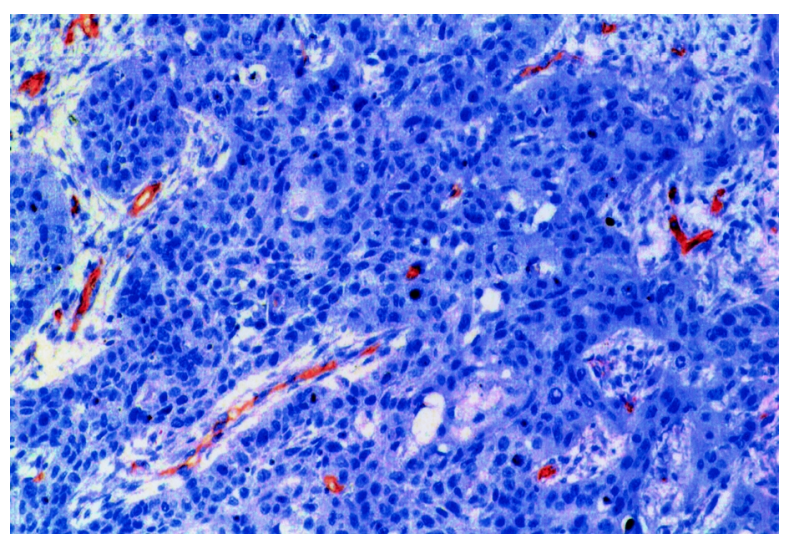

Figura 2 - Densidade microvascular no sítio primário e nas metástases linfonodais

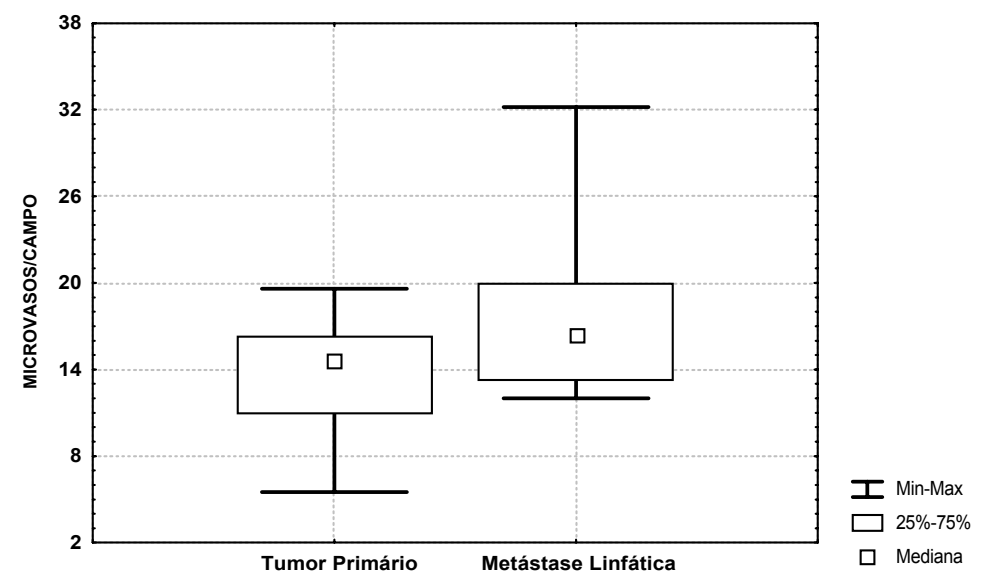

Figura 3 - Relação entre a densidade microvascular no sítio primário e nas respectivas metástases

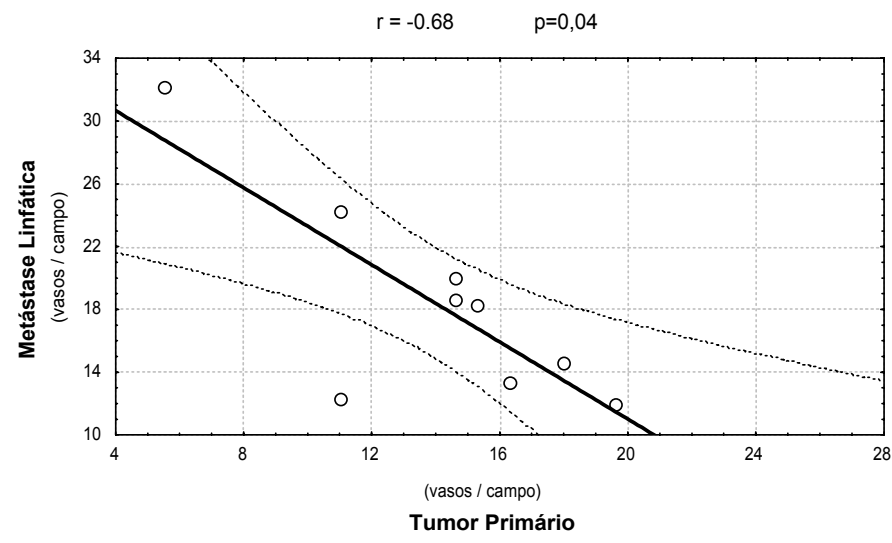


Amar A et al.

\begin{tabular}{lcc}
\hline \multicolumn{3}{c}{ Tabela I - Densidade microvascular no tumor primário } \\
\hline NECROSE & Mediana & $\mathrm{Q}_{25-75 \%}$ \\
Presente & 15,3 & $11,0-19,6$ \\
Ausente & 15,6 & $14,6-18,1$ \\
INFILTRADOINFLAMATÓRIO & & \\
Discreto & 15,5 & $11,0-16,3$ \\
Intenso & 15,3 & $14,6-18,1$ \\
DESMOPLASIA & & \\
Discreta & 16,5 & $11,0-18,1$ \\
Intensa & 15,3 & $13,9-18,1$ \\
DIFERENCIAÇÃO & & $14,6-19,6$ \\
Graul & 15,0 & $13,3-18,0$ \\
$\quad$ Grau II- III & 15,6 & \\
\hline
\end{tabular}

Figura 4 - Densidade microvascular no tumor primário em relação ao estadiamento $T$

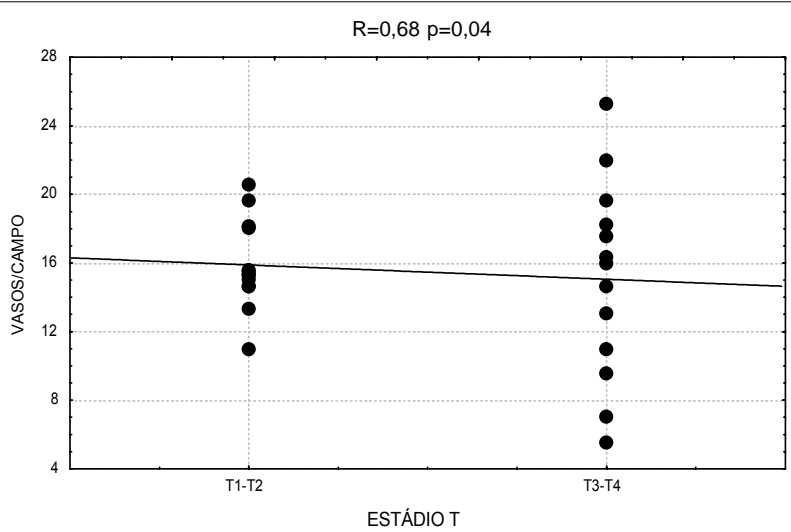

Figura 5 - Densidade microvascular no tumor primário na presença de metástase

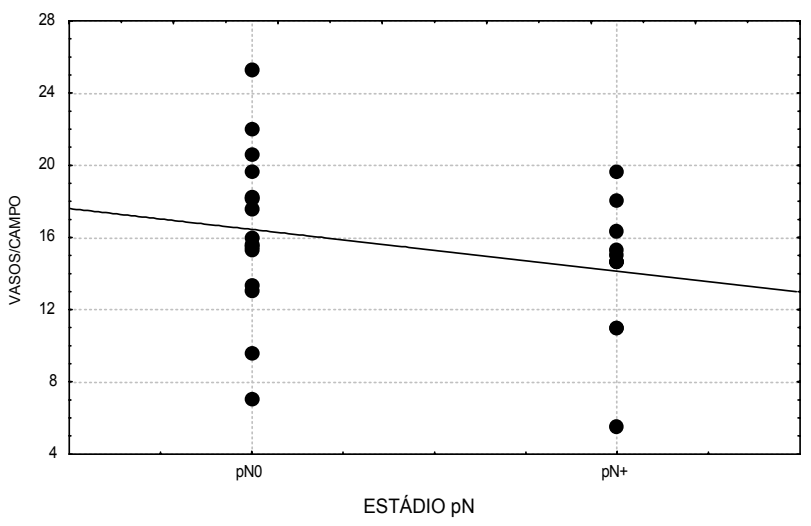

campo (7 a 25,3) no tumor primário e os tumores metastáticos ( $\mathrm{pN}+$ ) apresentaram mediana de 14,6 vasos/campo $(5,5$ a 19,6), $\mathrm{p}=0,12$ (Figura 5). sião da última consulta, com medianas de I5, I vasos/campo (5,5 a 20,6) e I 5,8 vasos/ campo $(9,6$ a 25,3$)$ respectivamente, $p=0,47$ (Figura 6). O seguimento dos pacientes assintomáticos apresentou média de 46 meses, com o mínimo de 18 meses.

\section{Discussão}

A relação inversa entre a densidade microvascular no sítio primário e nas metástases linfonodais sugere a existência de mecanismos de controle da disseminação da neoplasia maligna para o pescoço (regional) ou órgãos à distância (sistêmica). Modelos matemáticos explicam a ação mais significativa dos inibidores da angiogênese nos sítios distantes do tumor, em parte devido à meia-vida mais longa destas substâncias ${ }^{7}$. Em modelo experimental, foi confirmado o efeito supressor e esclerosante das irradiações sobre a angiogênese tanto na lesão primária quanto nas metástases linfonodais ${ }^{8}$. Não foi encontrada diferença significativa na densidade microvascular entre tumores metastáticos e nãometastaticos, semelhante ao observado por Leedy et a $\left.\right|^{4}$. Isto contrasta com o resultado de outros estudos, em que é relatado maior potencial de metastatização nos tumores mais vascularizados ${ }^{3,9}$. A disseminação metastática depende de diversos fatores além da angiogênese, pois a maior metastatização poderia ser contrabalançada pela inibição do desenvolvimento das metástases nos tumores primários mais angiogênicos. Williams et al. ${ }^{9}$, em pacientes com carcinoma epidermóide de boca sem metástases linfonodais clinicamente identificáveis, relataram maior índice de recidivas regionais entre os pacientes com tumores primários mais angiogênicos ${ }^{9}$. A angiogênese no sítio primário poderia auxiliar na identificação dos pacientes com pescoço falso-negativo (ausência de metástase linfonodal clínica - $\mathrm{N}_{0} \mathrm{e}$ presença de metástase linfonodal histológica $\mathrm{pN}+$ ), supondo que os tumores mais angiogênicos possam manter as metástases linfonodais quiescentes, com maior probabilidade de exames clínicos falso-negativos. Cabe ressaltar que os linfonodos, no presente estudo foram submetidos à avaliação rotineira (2 cortes), com menor probabilidade de detecção das micrometástases. Neste contexto, a avaliação da angiogênese pode auxiliar a elucidar o significado clínico das micrometástases, fazendo a diferenciação entre focos neoplásicos 
Figura 6 - Densidade microvascular no tumor primário e recidiva da doença

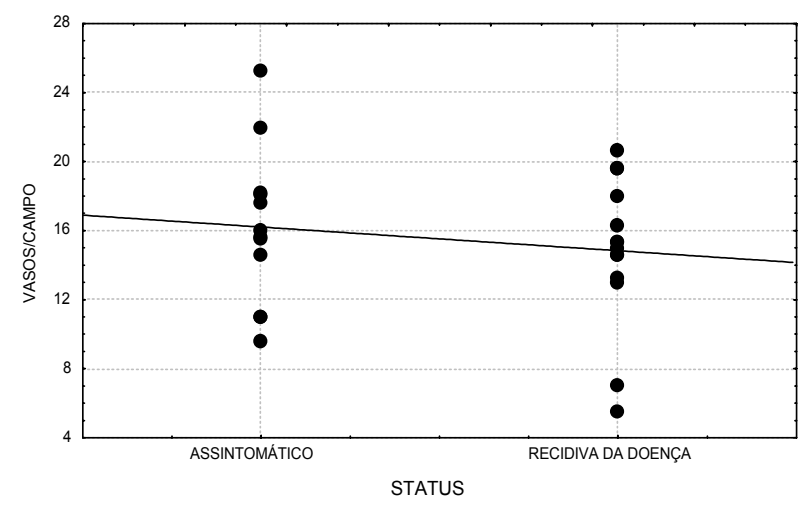

com crescimento suprimido e células "em trânsito". São necessárias amostras maiores para confirmação desta hipótesee para definição de um ponto de corte adequado, não se descartando a possibilidade da feitura de cortes seriados.

Diante de um possível efeito supressivo variável exercido pelo tumor primário, é difícil interpretar a angiogênese nas metástases. As medianas da densidade vascular foram semelhantes em ambos locais, embora as maiores densidades vasculares tenham sido observadas nas metástases (Figuras 2 e 3 ). Seria esperada maior propensão à metastatização entre os clones mais angiogênicos, mas a densidade vascular nas metástases pode refletir tanto as características dos clones originários como as diferenças no microambiente do linfonodo. A possibilidade de que metástases volumosas bem vascularizadas inibam a angiogênese no tumor primário também deve ser considerada.

As células CD34 positivas, que podem representar células dendríticas imaturas ou precursores endoteliais, têm sido encontradas em maior número no sangue periférico e no infiltrado inflamatório tumoral em portadores de câncer de cabeça e pescoço. Estas células causam imunossupressão local, podem estimular a angiogênese e têm sido associadas com pior prognóstico ${ }^{10-12}$. Embora estas células tenham sido identificadas no presente estudo, o CD34 é um marcador de membrana e o método imuno-histoquímico empregado não é adequado para a sua quantificação. Paradoxalmente, a necrose pode estar relacionada à maior angiogênese, o que seria explicado pela resposta inflamatória desencadeada por este tipo de morte celular. Leek et al ${ }^{13}$ apontam a presença de maior angiogênese em áreas adjacentes aos focos de necrose. A necrose, porém, também pode proporcionar a maturação das células dendríticas $\mathrm{CD} 34+$, diminuindo seus efeitos deletérios (enquanto imaturas) e proporcionando a apresentação antigênica ${ }^{14}$. No entanto, não foi observada relação entre a necrose, o infiltrado inflamatório e a densidade microvascular no presente estudo. Uma outra forma de avaliar a angiogênese consiste na mensuração da distância entre os vasos, que pode ser um indicador mais fidedigno da oxigenação tumoral do que a densidade vascular, mas esta variável não foi avaliada ${ }^{15}$. A relação de causa-efeito entre a necrose, a resposta inflamatória, a angiogênese e a proliferação do estroma ainda precisa ser esclarecida. As interleucinas e metaloproteinases que participam nestes processos também podem favorecer a invasão dos tecidos adjacentes pelas células neoplásicas, uma etapa importante no processo de metastatização $0^{16-18}$. A interação dinâmica entre estes fatores, porém, impõe a realização de estudos in vivo.

A angiogênese, isoladamente, não apresentou relação com a recidiva da doença em pacientes submetidos a tratamento cirúrgico. Para ter importância prognóstica individual, esta variável deveria apresentar resultado significativo mesmo em amostra pequena. Os resultados encontrados neste estudo sugerem que a angiogênese deva ser interpretada no contextoloco-regional.

\section{CONCLUSÕES}

I. Não houve relação significativa entre a densidade microvascular no tumor primário e as variáveis histológicas avaliadas (grau de diferenciação, padrão de invasão, infiltrado inflamatório, necrose e desmoplasia).

2. Não houve diferença significativa entre a densidade microvascular nos pacientes sem metástase linfonodal clínica ( $\mathrm{NN} 0$ ) e nos com metástase linfonodal histológica $(\mathrm{pN}+)$.

3. Não houve diferença significativa da densidade microvascular no período pós-operatório dos pacientes assintomáticos e nas recidivas.

4. Houve relação inversa da densidade microvascular no tumor primário e na respectiva metástase.

\section{SUMMARY}

MICROVASCULAR DENSITY IN CARCINOMA OF THE TONGUE

BACKGROUND. Assessment of microvascular density in squamous cell carcinoma of the oral tongue (primary lesion and metastasis).

METHODS. Immunohistochemical analysis by anti CD-34 of neoangiogenesis density and its relation with clinical and histological data concerning the prognosis. After optic microscopy amplification, the relation between microvascular density, clinicohistological data and prognosis, was established.

RESULTS. The microvascular density presented 15.4 vessels/field (5.5 to 25.3) in primary tumors and 16.4 vessels/field (I2 to 32.2) in lymph node metastases. It was observed an inverse relation between microvascular density in primarylesions and theirlymphnodemetastasis $(r=-0.68$ and $p=0,04)$.

CONCLUSIONS. No evidence was detected between microvascular density, histological features and prognosis.

There is an inverse relation between microvasculardensity in primary tumor and inlymphonode metastasis, suggesting a regional or systemic control of angiogenesis in squamous cell carcinoma of the oral tongue. [RevAssoc Med Bras 2002; 48(3): 204-8]

KeY Words: Cancer. Angiogenesis. Lymph node. Metastasis. Tongue. 
Amar A et al.

\section{REFERÊNCIAS}

I. Jones AS, Bin Hanafi Z, Nadapalan V. Do positive resection margins after ablative surgery for head and neck cancer adversaly affect prognosis? A study of 352 patients with recurrent carcinoma following radiotherapy treated by salvage surgery. $\mathrm{Br}$. J. Cancer, 1996; 76:128-2.

2. Zelefsky MJ, Harrison LB, Fass DE. Postoperative radiation therapy for squamous cell carcinomas of the oral cavity and oropharynx: impact of therapy on patients with positive surgical margins. Int J Radiation Oncology Biol Phys, 1993; 25: 17-21.

3. Ellis LM, Fidler IJ. Angiogenesis and metastasis. Eur J Cancer, 1996; 32: 245 I-60.

4. Leedy DA, Trune DR, KronzJD. Tumor angiogenesis, the 53 antigen, and cervical metastasis in squamous carcinoma of the tongue. Otolaryngol Head Neck Surg, 1 993; I I I: 4 I 7-22.

5. Gleich LL, Biddinger PW, Duperier FD. Tumor angiogenesis as a prognostic indicator in T2-T4 oral cavity squamous cell carcinoma: $A$ clinico-pathologic correlation. Head Neck, 1997; 19: 276-80.

6. Shpitzer T, Chaimoff M, Stern Y. Tumor angiogenesis as a prognostic factor in early oral tongue cancer. Arch Otolaryngol Head Neck Surg, 1996; I 22: 865-8.

7. Ramanujan S, Koenig GC, Padera TP. Local imbalance of proangiogenic and antiangiogenic factors: A potential mechanism of focal necrosis and dormancy in tumors. Cancer Res, 2000; 60:1442-8.

8. Camphausen K, Moses MA, Beecken WD. Radiation therapy to a primary tumor accelerates metastatic growth in mice. Cancer Res, 200I; 6I: 2207-II.

9. Williams JK, Carlson GW, Cohen C. Tumor angiogenesis as a prognostic fator in oral cavity tumors. Am J Surg, 1994; 168: 373-380.

10. Lathers DMR, Lubbers E, Wright MA. Dendritic cell differentiation pathways of CD34+ cells from the peripheral blood of head and neck cancer patients. J Leukoc Biol, 1999; 65: 623-8.

II. Young MRI, Wright MA, Lozano Y. Increased recurrence and metastasis in patients whose primary head and neck squamous cell carcinomas secreted granumocyte-macrophage colony-stimulating factor and contained CD34+ natural supressor cells. Int J Cancer, 1997; 74: 69-74.

12. Peichev M, Naiyer AJ, Pereira D. Expression of VEGFR-2 and $\mathrm{ACI} 33$ by circulating human CD34+ cells identifies a population of functional endothelial precursors. Blood, 2000;95: 952-8.

13. Leek RD, Landers RJ, Harris AL. Necrosis correlates with high vascular density and focal macrophage infiltration in invasive carcinoma of the breast. Br J Cancer, 1999;79: 99।-5.

14. Sauter B, Albert ML, Loise F. Consequences of cell death: Exposure to necrotic tumor cells, but not primary tissue cells or apoptotic cells, induces the maturation of immunostimulatory dendritic cells. J Exp Med, 2000; 191: 423-33.

15. West CML, Cooper RA, Loncaster JA. Tumor vascularity: A histological measure of angiogenesis and hypoxia. Cancer Res, 200I; 6I: 2907-I0.

16. Eisma RJ, Spiro JD, Kreutzer DL. Role of angiogenic factors: Coexpression of interleukin- 8 and vascular endothelial growth factor in patients with head and neck squamous carcinoma. Laryngoscope, 1999; 109: 687-93.

17. Polverini PJ. How the extracellular matrix and macrophages contribute to angiogenesisdependent diseases. Eur J Cancer, 1996; 32: 2430-37.

18. Moore BB, Keane MP, Addison CL. CXC chemokine modulation of angiogenesis: The importance of balance between angiogenic and angiostatic members of the family. J Invest Med, 1998; 46: II4-20.

Artigo recebido: 17/09/2001 Aceito para publicação: | 4/03/2002 